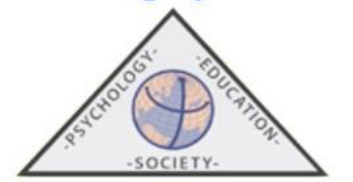

\title{
Comprensión lectora en autismo: revisión de las intervenciones y sus niveles de evidencia
}

\author{
Miriam Rivero-Contreras y David Saldaña \\ Universidad de Sevilla \\ Recibido el 23 de diciembre de 2019. Aceptado el 21 de abril de 2021
}

RESUMEN: El objetivo del presente estudio fue determinar qué estrategias de intervención en comprensión lectora se han evaluado específicamente en personas con autismo, su impacto en la comprensión lectora, y su calidad metodológica. Para ello, se analizaron revisiones presentes en la literatura científica hasta 2019, y se realizó una búsqueda de estudios primarios en bases de datos entre 2020 y febrero de 2021. En la primera búsqueda se encontraron 35 trabajos, de los cuales solo tres eran revisiones. Además, otra revisión fue identificada en las referencias bibliográficas. En estas cuatro revisiones fueron seleccionados 22 estudios primarios para su análisis. En cuanto a la segunda búsqueda, se identificaron 27 estudios, de los cuales fueron seleccionados uno. Un estudio primario fue incluido a partir de la búsqueda en las referencias bibliográficas. Por tanto, un total de 24 estudios primarios fueron seleccionados para su análisis. Los resultados del análisis mostraron diferentes tipos de instrucción en comprensión lectora, las cuales aumentaban la comprensión lectora en personas con autismo, pero su calidad metodológica resultó variada. La mayor parte de los estudios experimentales presentaban un nivel de evidencia fuerte, mientras que los estudios de caso único tenían un nivel de evidencia moderado. En conclusión, son relativamente pocos los estudios que se han desarrollado hasta el momento y su calidad metodológica es variada en función del tipo de estudio desarrollado.

Palabras clave: Comprensión del texto; intervención; metodología; autismo.

\section{Reading comprehension in autism: review of interventions and their levels of evidence}

\begin{abstract}
The aim of this study was to determine which intervention strategies in reading comprehension have been specifically evaluated in people with autism, their impact on reading comprehension, and their methodological quality. We analyzed primary studies located in reviews up to 2019 , and primary studies located in databases from 2020 to February 2021. In the first search 35 items were found, of which only three were reviews. In addition, another review was identified in the bibliographic references. From these four reviews, 22 primary studies were selected for analysis. Twenty-seven primary studies from the years 2020 and 2021 were identified, from which one was selected. One primary study was included from the search in the bibliographic references. Therefore, a total of 24 primary studies were selected for analysis. The results of the analyses showed different types of instruction in reading comprehension, which improved reading comprehension in people with autism, but their methodological quality was varied. Most of the experimental studies had a strong level of evidence, whereas the single case studies had a moderate level of evidence. In conclusion, a relatively limited number of studies have been developed so far and their methodological quality is varied.
\end{abstract}

Keywords: Text comprehension; intervention; methodology; autism. 


\section{Introducción}

\section{Comprensión lectora y autismo}

El trastorno del espectro del autismo (TEA) es un trastorno del neurodesarrollo que se caracteriza por la presencia de dificultades persistentes en la comunicación e interacción social en varios contextos, y por la presencia de patrones restrictivos y repetitivos de comportamiento, intereses o actividades (American Psychiatric Association, 2013). Aunque entre los criterios diagnósticos del TEA la alteración en el lenguaje no consta como criterio específico, es frecuente encontrar comorbilidad con los trastornos del lenguaje. Las dificultades en el lenguaje pueden afectar tanto al lenguaje oral como al escrito, colocando a las personas con autismo en alto riesgo de fracaso académico. En el caso del lenguaje escrito, hasta hace unos años, se había constatado un perfil específico de dificultades de comprensión en una alta proporción de niños y niñas con TEA (Henderson et al., 2014; Nation et al., 2006; Nation y Norbury, 2005). Así, Brown et al. (2013) revisaron 36 estudios en los que se comparaban la comprensión lectora de personas con TEA y la de un grupo control, es decir, sin ninguna dificultad o trastorno. En su meta-análisis encontraron una puntuación media en el grupo de autismo 0.7 desviaciones tipo inferior al grupo control. Este dato es consistente con el hallazgo de Nation et al. (2006) de que un $32 \%$ de los niños y niñas con autismo presentaba una discrepancia de al menos una desviación tipo entre sus puntuaciones de comprensión y decodificación, aunque esta fuera adecuada.

Sin embargo, en trabajos recientes se han observado varios perfiles diferenciados según la severidad de las dificultades de comprensión (Davidson y Ellis Weismer, 2014; McIntyre et al., 2017; Solari et al., 2019). El primer perfil se corresponde con una ausencia de déficit en la lectura de palabras y en la comprensión auditiva/lectora. El segundo y tercer perfil es aquel que presenta un déficit de comprensión en ausencia de déficit en la lectura de palabras; concretamente, habilidades de comprensión relativamente más bajas (pero no necesariamente por debajo del promedio), y habilidades de comprensión por debajo del promedio en estos dos perfiles respectivamente. Y el cuarto y quinto perfil se corresponden con un déficit mixto en los que tanto la lectura como la comprensión de palabras está afectada (para más información, véase Davidson, 2021). Por el momento, solo un estudio longitudinal ha constatado la estabilidad de cuatro de los cinco perfiles, siendo el segundo perfil el único no confirmado (Solari et al., 2019).

\section{El proceso de lectura}

La lectura es una actividad compleja que implica un doble proceso de decodificación y comprensión. La decodificación consiste en el reconocimiento eficaz de las palabras a partir del uso de reglas de correspondencia grafema-fonema. Para ello, el lector debe ser capaz de derivar la representación de la palabra a la entrada del léxico mental (unidad de conocimiento interiorizado del vocabulario), y así poder recuperar su información semántica. En cuanto a la comprensión del lenguaje oral, la persona debe ser capaz de tomar dicha información semántica y realizar interpretaciones y narraciones (Hoover y Gough, 1990).

Por otro lado, la comprensión lectora es el resultado de un proceso multinivel, que va desde la identificación de los grafemas hasta considerar el texto como un todo. El modelo más importante y conocido de comprensión lectora es el modelo de construcción-integración de Kintsch (1998). Según este autor, se dan dos tipos de representaciones mentales cuando leemos: la representación o base textual (construcción) y la representación situacional o modelo de situación (integración). Para la elaboración de la representación o base textual, en primer lugar, se elaboran proposiciones como estructuras básicas de significado. Después, estas proposiciones se unen para formar la microestructura como nivel 
semántico superficial para establecer la coherencia local del texto. Y la unión de estas microestructuras forman la llamada macroestructura como nivel semántico profundo que determina la coherencia global del texto. Por tanto, estamos hablando de un procesamiento ascendente de carácter semánticoproposicional. Por otro lado, en la elaboración de la representación situacional o modelo de situación se establece un modelo mental que permite integrar la base textual con los conocimientos previos del lector, resultando en un procesamiento descendente de carácter episódico.

La discrepancia que observamos en el autismo entre decodificación y comprensión es consistente con el modelo simple de lectura (Gough y Tunmer, 1986). Según este modelo, la comprensión lectora es el resultado de dos componentes: la decodificación y la comprensión del lenguaje oral, ambos de igual importancia. Los mismos autores del modelo desarrollaron una ecuación para explicar la relación entre comprensión lectora ("R”), decodificación ("D”) y comprensión oral ("C"):

$$
\mathrm{R}=\mathrm{D} \text { X C }
$$

Los valores de dichos componentes pueden oscilar de 0 a 1 , por lo que, si se da ausencia de decodificación o de comprensión oral, la comprensión lectora se ve afectada. Así, tanto la decodificación como la comprensión oral son componentes necesarios para la comprensión de la lectura.

Un modelo más reciente, el modelo de efecto indirecto y directo de lectura (Kim, 2017), basado en el modelo simple de lectura y que integra aspectos del modelo de construcción-integración, sostiene que la comprensión lectora es el resultado de la decodificación y la comprensión del lenguaje oral (efecto directo), pero va más allá, identificando variables que influyen en la comprensión lectora como la memoria de trabajo, el vocabulario, la morfosintaxis, la inferencia, el monitoreo de la comprensión y la teoría de la mente (efecto indirecto), y que funcionan como predictoras de la comprensión lectora.

Aunque muchas personas con TEA presentan dificultades en el componente de decodificación, como hemos señalado más arriba, se observa un perfil específico de dificultades en la comprensión que va más allá de la lectura de palabras (Brown et al., 2013). Por tanto, parece necesario contar con intervenciones educativas apropiadas para abordar estas dificultades de comprensión en concreto.

\section{Instrucción en comprensión lectora}

El Panel Nacional de Lectura (2000) propuso numerosas estrategias de aprendizaje a partir de cinco elementos esenciales: conciencia fonológica, fonética, fluidez lectora, vocabulario y comprensión lectora. Los tres primeros se centran en desarrollar la decodificación, mientras que los dos últimos están enfocados al desarrollo y la comprensión del lenguaje.

Perfetti et al. (2005) identificaron tres componentes esenciales en la comprensión lectora: sensibilidad a la estructura de la historia, inferencia y monitoreo de la comprensión, siendo estos dos últimos importantes fuentes tanto de desarrollo como de problemas de comprensión. En esta línea y de acuerdo con varias revisiones sistemáticas en este campo (El Zein et al., 2014; Khowaja y Salim, 2013; Tárraga-Mínguez et al., 2020), la instrucción en comprensión lectora puede incorporar diversas técnicas, como pueden ser monitorear (explicación de palabras desconocidas, realización de inferencias, uso de organizadores gráficos, discusiones continuas durante la lectura), estructurar la historia (identificar las ideas principales y las secundarias), generar y responder a preguntas (evaluación del conocimiento lector, organizar ideas), resumir (organizar la información), entre otras.

Aunque existe una amplia literatura sobre las intervenciones en comprensión lectora en niños y niñas sin otros trastornos en el desarrollo (Hulme y Snowling, 2011), no está claro que sean de aplicación para las personas con autismo con el mismo tipo de dificultades lectoras. El objetivo del presente estudio es determinar qué estrategias de intervención en comprensión lectora se han evaluado específicamente en la población de personas con TEA, cuál es el grado de impacto que las diferentes 
estrategias tienen sobre la comprensión de los lectores con autismo, y la calidad metodológica de estas investigaciones.

\section{Metodología}

Se realizó una búsqueda en las bases de datos ERIC, Mendline y PsycINFO. En primer lugar, se llevó a cabo una primera búsqueda con el objetivo de localizar estudios de revisión teórica o sistemática desarrollados hasta el momento. Después, se realizó una segunda búsqueda de estudios primarios comprendidos entre 2020 y febrero de 2021, puesto que la última revisión encontró estudios hasta 2019. Además, hubo una tercera búsqueda de estudios en las referencias bibliográficas de los estudios finalmente seleccionados.

Tanto para la primera búsqueda como para la segunda se utilizaron tres bloques de palabras clave. El primer bloque de palabras sinónimas estaba relacionado con las diferentes denominaciones de autismo, el segundo con la comprensión lectora, y el tercero con el de intervención, además de la palabra "review" o "synthesis" para la primera búsqueda. El primer bloque contenía las palabras "autism spectrum disorders, "child development disorders", "pervasive developmental disorders" y "autism"; el segundo bloque, las palabras "reading comprehension", y en el tercer bloque se utilizaron "treatment", "therapy", "intervention", "methods", "teaching methods", "reading skills", "response to intervention", "training" y "education".

Siguiendo el esquema PICO (Hoffmann et al., 2014), se definieron los siguientes criterios de inclusión (además de las fechas indicadas arriba): los participantes deberían ser personas con autismo (Población), objeto de una intervención de naturaleza psicoeducativa (Intervención), evaluada a través de cualquier estudio experimental, cuasi-experimental o de caso único, sin necesidad de grupo control específico (Control), centrado en la mejora de la comprensión lectora (Resultado). Solo se incluyeron publicaciones en lengua inglesa. Se aceptaron tanto tesis doctorales publicadas como artículos en revistas con revisión por pares, y se excluyeron estudios comparativos de intervenciones. Como se ha señalado más arriba, en la primera fase se localizaron las revisiones realizadas hasta febrero de 2021, y se extrajeron los estudios primarios descritos en las mismas. En la segunda se detectaron directamente los estudios primarios.

En la primera búsqueda se obtuvieron 35 resultados, de los cuales solo tres revisiones cumplían los criterios de inclusión (El Zein et al., 2014; Khowaja y Salim, 2013; Tárraga-Mínguez et al., 2020). La cuarta revisión seleccionada se encontró en las referencias bibliográficas de dichas revisiones (Whalon et al., 2009). Una vez analizadas las cuatro revisiones, se escogieron 22 estudios que cumplían con los criterios de inclusión. Además, se escogió un estudio más tras la búsqueda en las referencias bibliográficas de los estudios seleccionados.

En la búsqueda de estudios de 2020 a febrero de 2021 se obtuvieron 27 resultados, de los cuales solo uno cumplía los criterios de inclusión. No se escogieron más estudios tras la búsqueda en las referencias bibliográficas de los estudios seleccionados por no cumplir ninguno los criterios de inclusión (ver Figura 1). Por tanto, se han incluido en esta revisión un total de 24 estudios que aplicaban una intervención en comprensión lectora en personas con autismo.

La evaluación de los indicadores de calidad metodológica de todos los estudios incluidos fue llevada a cabo por los dos autores de forma independiente, uno de los cuales cuenta con una amplia experiencia en programas de comprensión lectora en autismo. Los desacuerdos se resolvieron por consenso. 


\section{Figura 1}

Esquema del proceso de selección e inclusión de los estudios primarios

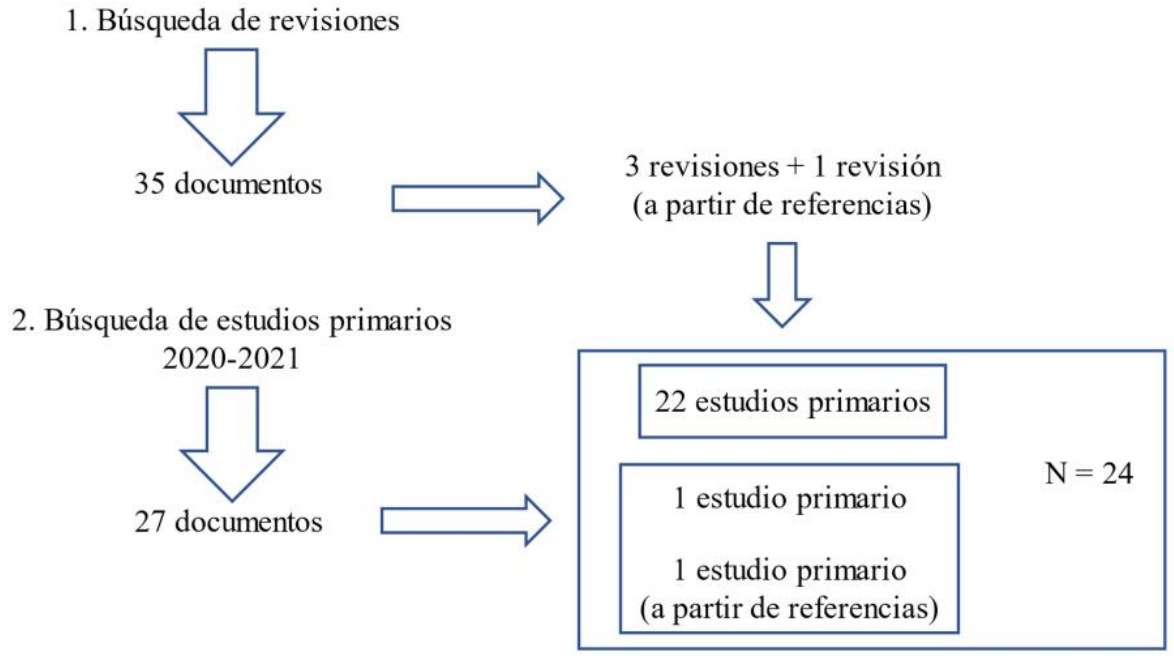

\section{Resultados}

\section{Tipos de instrucción en comprensión lectora y resultados encontrados}

Se identificaron los siguientes cinco grupos de intervenciones: 1) instrucción de estrategias, 2) indicación de anáforas, 3) instrucción explícita, 4) grupos de estudiantes y 5) instrucción combinada.

En la instrucción en estrategias se enseña una serie de estrategias que han demostrado ser útiles para aumentar la comprensión lectora como la generación de preguntas, la realización de organizadores gráficos o hacer predicciones. La indicación de anáforas consiste en identificar referentes en el texto para así poder facilitar la comprensión lectora. La instrucción explícita aplica diferentes estrategias como modelado, explicaciones y práctica supervisada. El cuarto grupo se basa en mejorar la comprensión lectora a través de grupos de estudiantes que trabajan juntos. Y la intervención combinada consiste en aplicar dos tipos de intervención: la instrucción de estrategias y la instrucción explícita. En la mayoría de los estudios los participantes con TEA pudieron mejorar la comprensión lectora tras las intervenciones, excepto aquellos a los que les fueron aplicados el programa de lectoescritura ABRACADABRA en el entorno escolar (Arciuli y Bailey, 2019) perteneciente al grupo de intervención instrucción de estrategias (véase el Apéndice para una descripción completa de los estudios primarios y las intervenciones).

\section{Solidez de la evidencia empírica: calidad metodológica}

A pesar de los buenos resultados obtenidos en todos los estudios, la calidad metodológica resultó ser muy variada. Para poder valorar la evidencia de cada estudio, se utilizó la clasificación de Reichow et al. (2008). Estos autores establecen una serie de criterios tanto para estudios experimentales como de caso único (véase la Tabla 1). Según su modelo, un estudio con evidencia fuerte sería el que cumple con todos los indicadores primarios y cuatro de los secundarios (o tres en los estudios de caso). 
Las investigaciones con evidencia adecuada cumplirían cuatro de los indicadores primarios y dos secundarios; los de evidencia débil cuatro indicadores primarios y menos de dos secundarios.

Tabla 1

Indicadores primarios y secundarios de calidad según tipo de estudio (Reichow et al., 2008)

\begin{tabular}{|c|c|c|c|}
\hline \multicolumn{2}{|c|}{ Experimental } & \multicolumn{2}{|c|}{ Caso único } \\
\hline Primarios & Secundarios & Primarios & Secundarios \\
\hline $\begin{array}{l}\text { Características de los } \\
\text { participantes }(\mathrm{P})\end{array}$ & $\begin{array}{l}\text { Asignación aleatoria } \\
\text { (A) }\end{array}$ & $\begin{array}{l}\text { Características de } \\
\text { los participantes }(\mathrm{P})\end{array}$ & $\begin{array}{l}\text { Acuerdo } \\
\text { interobservadores (AI) }\end{array}$ \\
\hline $\begin{array}{l}\text { Variable Independiente } \\
(\mathrm{VI})\end{array}$ & $\begin{array}{l}\text { Acuerdo } \\
\text { interobservadores (AC) }\end{array}$ & $\begin{array}{l}\text { Variable } \\
\text { independiente (VI) }\end{array}$ & Kappa (K) \\
\hline $\begin{array}{l}\text { Condición de comparación } \\
\text { (C) }\end{array}$ & $\begin{array}{l}\text { Condición de ciego } \\
\text { (Ci) }\end{array}$ & $\begin{array}{l}\text { Condición de la } \\
\text { línea base (LB) }\end{array}$ & $\begin{array}{l}\text { Condición de ciego } \\
\text { (Ci) }\end{array}$ \\
\hline $\begin{array}{l}\text { Variable dependiente } \\
\text { (VD) }\end{array}$ & $\begin{array}{l}\text { Fidelidad al } \\
\text { tratamiento }(\mathrm{F})\end{array}$ & $\begin{array}{l}\text { Variable } \\
\text { dependiente (VD) }\end{array}$ & $\begin{array}{l}\text { Fidelidad al } \\
\text { tratamiento }(\mathrm{F})\end{array}$ \\
\hline $\begin{array}{l}\text { Enlace entre pregunta de } \\
\text { investigación y análisis de } \\
\text { datos (PD) }\end{array}$ & $\begin{array}{l}\text { Mortandad } \\
\text { experimental (ME) }\end{array}$ & $\begin{array}{l}\text { Análisis visual } \\
(\mathrm{AV})\end{array}$ & $\begin{array}{l}\text { Generalización y/o } \\
\text { mantenimiento (GM) }\end{array}$ \\
\hline $\begin{array}{l}\text { Usos de los tests } \\
\text { estadísticos }(\mathrm{T})\end{array}$ & $\begin{array}{l}\text { Generalización y/o } \\
\text { mantenimiento (GM) }\end{array}$ & $\begin{array}{l}\text { Control } \\
\text { experimental (CE) }\end{array}$ & Validez social (VS) \\
\hline & $\begin{array}{l}\text { Tamaño del efecto } \\
\text { (TE) }\end{array}$ & & \\
\hline
\end{tabular}

A continuación, se muestran los indicadores primarios y secundarios de cada estudio y su grado de evidencia (véanse Tablas 2 y 3):

Tabla 2

Indicadores primarios y secundarios de calidad y evidencia de los estudios experimentales

\begin{tabular}{|c|c|c|c|c|c|c|c|c|c|c|c|c|c|c|c|}
\hline \multirow[b]{2}{*}{ Estudio } & \multicolumn{6}{|c|}{ Indicadores primarios } & \multicolumn{8}{|c|}{ Indicadores secundarios } & \multirow[b]{2}{*}{ Evidencia } \\
\hline & $\mathrm{P}$ & VI & $\mathrm{C}$ & VD & $\mathrm{PD}$ & $\mathrm{T}$ & A & $\mathrm{AC}$ & $\mathrm{Ci}$ & $\mathrm{F}$ & $\mathrm{ME}$ & GM & $\mathrm{TE}$ & VS & \\
\hline \multicolumn{16}{|c|}{ Identificación anafórica } \\
\hline A & Parcial & Sí & Sí & Sí & Sí & Sí & Sí & Sí & Sí & No & Sí & No & Sí & Sí & Adecuada \\
\hline \multicolumn{16}{|c|}{ Instrucción de estrategias } \\
\hline B & Sí & Sí & Sí & Sí & Sí & Sí & Sí & Sí & No & Sí & Sí & No & Sí & Sí & Fuerte \\
\hline $\mathrm{C}$ & Parcial & Sí & No & Sí & Sí & Sí & No & Sí & No & No & Sí & No & No & Sí & Adecuada \\
\hline $\mathrm{D}$ & Sí & Sí & Sí & Sí & Sí & Sí & Sí & No & No & No & Sí & No & Sí & Sí & Fuerte \\
\hline \multicolumn{16}{|c|}{ Combinada } \\
\hline $\mathrm{E}$ & Sí & Sí & Sí & Sí & Sí & Sí & Sí & Sí & Sí & Sí & Sí & Sí & Sí & Sí & Fuerte \\
\hline $\mathrm{F}$ & Parcial & Sí & Sí & Sí & Sí & Sí & No & No & No & No & Sí & No & Sí & Sí & Débil \\
\hline
\end{tabular}


Nota. Los estudios experimentales analizados fueron los siguientes: el estudio A desarrollado por O’Connor y Klein (2004); el estudio B desarrollado por Arciuli y Bailey (2019); el estudio C desarrollado por Asberg y Sandberg (2010); el estudio D desarrollado por Bailey et al. (2017); el estudio E desarrollado por Roux et al. (2015); y el estudio F desarrollado por Turner et al. (2017).

Tabla 3

Indicadores primarios y secundarios de calidad y evidencia de los estudios de caso único

\begin{tabular}{|c|c|c|c|c|c|c|c|c|c|c|c|c|c|}
\hline \multirow{2}{*}{ Estudio } & \multicolumn{6}{|c|}{ Indicadores primarios } & \multicolumn{6}{|c|}{ Indicadores secundarios } & \multirow{2}{*}{ Evidencia } \\
\hline & $\mathrm{P}$ & VI & VD & LB & $\mathrm{AV}$ & $\mathrm{CE}$ & AI & $\mathrm{K}$ & $\mathrm{F}$ & $\mathrm{Ci}$ & GM & VS & \\
\hline \multicolumn{14}{|c|}{ Instrucción de estrategias } \\
\hline A & Parcial & Sí & Sí & Sí & Sí & Sí & Sí & No & No & No & Sí & Sí & Adecuada \\
\hline $\mathrm{B}$ & Sí & Sí & Sí & Sí & Sí & Sí & Sí & No & Sí & No & Sí & Sí & Fuerte \\
\hline $\mathrm{C}$ & Parcial & Sí & Sí & Sí & Sí & Sí & Sí & No & Sí & No & Sí & Sí & Adecuada \\
\hline $\mathrm{D}$ & Parcial & Sí & Sí & Sí & Sí & Sí & Sí & No & Sí & No & Sí & Sí & Adecuada \\
\hline $\mathrm{E}$ & Sí & Sí & Sí & Sí & Sí & Sí & Sí & Sí & Sí & No & No & Sí & Fuerte \\
\hline $\mathrm{F}$ & Sí & Sí & Sí & Sí & Sí & Sí & Sí & No & Sí & No & Sí & Sí & Fuerte \\
\hline \multicolumn{14}{|c|}{ Instrucción explícita } \\
\hline $\mathrm{G}$ & Sí & Sí & Sí & Sí & Sí & No & Sí & No & Sí & No & Sí & Sí & Adecuada \\
\hline $\mathrm{H}$ & Parcial & Sí & Sí & Sí & Sí & Sí & Sí & No & Sí & No & Sí & Sí & Adecuada \\
\hline $\mathrm{I}$ & Parcial & Sí & Sí & Sí & Sí & Sí & Parcial & No & Sí & No & Sí & Sí & Adecuada \\
\hline \multicolumn{14}{|c|}{ Combinada } \\
\hline $\mathrm{J}$ & Parcial & Sí & Sí & Sí & No & Sí & Sí & No & Sí & No & Sí & Sí & Adecuada \\
\hline $\mathrm{K}$ & Sí & Sí & Sí & Sí & Sí & Sí & Sí & No & Sí & No & No & Sí & Fuerte \\
\hline $\mathrm{L}$ & Parcial & Sí & Sí & Sí & Sí & Sí & Sí & No & Sí & No & Sí & Sí & Adecuada \\
\hline M & Parcial & Sí & Sí & Sí & Sí & Sí & Sí & No & Sí & No & Sí & Sí & Adecuada \\
\hline $\mathrm{N}$ & Sí & Sí & Sí & No & No & No & Sí & No & Sí & No & No & Sí & Débil \\
\hline \multicolumn{14}{|c|}{ Grupo de estudiantes } \\
\hline $\mathrm{O}$ & Sí & Sí & Sí & Sí & Sí & No & Sí & No & No & No & No & Sí & Adecuada \\
\hline $\mathrm{P}$ & Sí & Sí & No & Sí & Sí & No & Sí & No & No & No & No & Sí & Adecuada \\
\hline Q & Sí & Sí & No & Sí & Sí & No & Sí & No & No & No & No & Sí & Adecuada \\
\hline $\mathrm{R}$ & Sí & Sí & Sí & Sí & Sí & Sí & Sí & No & Sí & No & Sí & Sí & Fuerte \\
\hline
\end{tabular}

Nota. Los estudios de caso único analizados fueron los siguientes: el estudio A desarrollado por Bethune y Wood (2013); el estudio B desarrollado por Browder et al. (2017); el estudio C desarrollado por Carnahan et al. (2016); el estudio D desarrollado por Stringfield et al. (2011); el estudio E desarrollado por Whalon y Handline (2008); el estudio F desarrollado por Williamson et al. (2015); el estudio G desarrollado por Flores y Ganz (2007); el estudio H desarrollado por Head et al. (2018); el estudio I desarrollado por Howorth et al. (2016); el estudio J desarrollado por Drill y Bellini (2021); el estudio K desarrollado por Jackson y Handline (2020); el estudio L desarrollado por Kim et al. (2018); el estudio M desarrollado por Singh et al. (2017); el estudio N desarrollado por Solis et al. (2021); el estudio O desarrollado por Kamps et al. (1989); el estudio P desarrollado por Kamps et al. (1994); el estudio Q desarrollado por Kamps et al. (1995); el estudio R desarrollado por Reutebuch et al. (2015) 
Como se puede observar en las anteriores tablas, seis estudios son experimentales, mientras que el resto son de caso único. La mayoría de las investigaciones presenta una evidencia adecuada, y algunos, evidencia fuerte, debido a que cuentan con bastantes indicadores primarios (un mínimo de cuatro) y algunos secundarios (un mínimo de dos). Aunque si nos fijamos en el tipo de estudio, la mitad de los estudios experimentales presentan un nivel de evidencia fuerte en comparación con los estudios de caso único, de los cuales una gran parte tiene un nivel de evidencia adecuada.

Respecto a los estudios experimentales, es llamativo el incumplimiento de los indicadores de ciego y generalización y/o mantenimiento en cuatro y cinco de los seis estudios. Asimismo, la mayoría no suelen cumplir el indicador de fidelidad al tratamiento.

En cuanto a los estudios de caso único, solo uno calcula el coeficiente Kappa, y parte de ellos no poseen control experimental ni tampoco registran los datos para observar la generalización y/o mantenimiento de la intervención. Además, ningún estudio cumplió el indicador de ciego. Un punto a favor que tienen es el acuerdo interobservadores, los cuales presentan valores iguales o superiores a .80 en la mayoría de los trabajos. Asimismo, la mayoría contiene una línea base con tres o más puntos de medida, la cual ofrece el punto de partida de los participantes, y muestra gráficos con los datos del rendimiento de cada uno de ellos.

Por otro lado, todos los estudios presentan indicadores validez social. Esto significa que son investigaciones realizadas en contextos naturales, sus variables dependientes son socialmente importantes, las intervenciones son a tiempo y costo efectivas, y se tienen en cuenta las opiniones de los participantes y/o sus padres, entre otros aspectos. Por ende, esto hace que sea un aspecto relevante a tener en cuenta en el desarrollo y la validación de intervenciones.

\section{Discusión}

El principal objetivo de este trabajo era determinar el tipo de estrategias de intervención desarrolladas, el impacto de dichas intervenciones y su grado de evidencia. Encontramos cuatro revisiones sistemáticas, de las cuales solo una realizaba un análisis de la calidad metodológica (El Zein et al., 2014). A diferencia de este estudio en el que se evaluó múltiples indicadores en función del tipo de investigación, esta revisión solo tomó en consideración cinco parámetros para evaluar la evidencia de una investigación. Estos parámetros fueron el control experimental, la variable dependiente definida operacionalmente, la descripción de la intervención, el acuerdo interobservadores y la fidelidad del tratamiento. En el presente trabajo, los tres primeros parámetros eran considerados indicadores primarios, mientras que los dos últimos eran indicadores secundarios.

En relación a los estudios encontrados, las dos revisiones sistemáticas más antiguas (El Zein et al., 2014; Khowaja y Salim, 2013), encontraron 11 y 12 estudios, mientras que la revisión más reciente (Tárraga-Mínguez et al., 2020), encontró 25 estudios. En esta revisión, se puede observar que continúan siendo relativamente pocas las publicaciones destinadas a evaluar la eficacia y/o efectividad de diferentes estrategias de intervención. En concreto, 24 estudios, uno menos que la revisión encontrada más reciente, puesto que se excluyeron estudios comparativos de intervenciones. Por tanto, llama la atención el reducido número de estudios encontrados, a pesar de conocerse la dificultad que presentan las personas con TEA en esta área y lo importante que es para el proceso de aprendizaje y el desarrollo cognitivo, social y afectivo de las personas.

Todos los estudios, excepto uno (Arciuli y Bailey, 2019), encontraron mejorías en la comprensión lectora tras aplicar su intervención. Además, destaca el uso de los organizadores gráficos en la mejora de los niveles de comprensión lectora. Y la intervención con grupo de estudiantes parece impactar positivamente no solo en la comprensión lectora, sino también en las interacciones sociales y conductas desafiantes, por lo que parece ser una línea prometedora de intervención. 
En cuanto a las características particulares de las personas con TEA, estas hacen referencia a las dificultades que tienen para extraer información del texto que se encuentra de forma implícita. Por tanto, las intervenciones de los estudios analizados parecen resultar útiles para obtener dicha información y poder desarrollar estrategias implicadas en la comprensión, como son la integración de ideas, la construcción de un significado global, el uso efectivo de los conocimientos previos a través de las inferencias, la explicación de palabras desconocidas, y diferentes estrategias metacognitivas como la planificación, evaluación y regulación. Todas estas estrategias están relacionadas con algunas de las variables predictoras del modelo de efecto indirecto y directo de lectura (Kim, 2017), como son la memoria de trabajo, el vocabulario, la inferencia y el monitoreo de la comprensión, las cuales resultan útiles para mejorar la comprensión lectora. Ahora bien, hay que tener en cuenta que en la mayor parte de los casos se trata de estrategias de uso general en el campo de la comprensión lectora, que han sido probadas con participantes con autismo. No son estrategias desarrolladas teniendo en cuenta los perfiles específicos de estos.

Otro punto a considerar es la calidad y tipo de los diseños de evaluación empleados. La mayoría presenta casos únicos, que permiten acercarse a la realidad de cada participante y sus dificultades, pero que reducen la posibilidad de extrapolar los resultados a la población con TEA. Asimismo, tanto en los trabajos experimentales como en los de caso único, el número de participantes es bajo. Nótese también que parte de los estudios no suele presentar fidelidad al tratamiento. Este indicador se refiere a la existencia en la investigación de mecanismos que garanticen que la intervención se ha aplicado correctamente, con una intensidad apropiada. Su cumplimiento es de suma importancia para saber que todos los implicados en la investigación han seguido los mismos pasos y que el cambio se debe a la intervención y no a otra variable externa.

La condición de ciego es un parámetro de evidencia difícil de conseguir, sobre todo si los evaluadores son los mismos que realizan las intervenciones, o si los evaluadores están presentes mientras que se llevan a cabo las estrategias. Sin embargo, no es incompatible con una intervención psicoeducativa, y los investigadores deberían introducir este mecanismo de prevención de sesgo.

Otro indicador que no suelen cumplir los trabajos (aunque se observa alguna mejoría en los más recientes) es la adecuada descripción de las características de los participantes (como edad, sexo y diagnóstico de los participantes) y de los interventores. Este segundo dato permite conocer el tipo de formación que tienen para realizar la intervención.

En cuanto a la fase que evalúa la generalización y/o mantenimiento de la intervención, solo la mitad de los estudios lo realizan. Su inclusión permitiría observar el efecto de la intervención a lo largo del tiempo.

En resumen, como exponen Brown et al. (2013), la pobre comprensión lectora es una dificultad presente en muchas personas con autismo, y son pocos los estudios que se han desarrollado hasta el momento, por lo que es de suma importancia continuar en la búsqueda de intervenciones eficaces de cara a mejorar la comprensión. Por otra parte, el grado de evidencia resulta en estos momentos moderado, pero sería deseable incrementar el volumen de estudios con un grado de evidencia fuerte. Además de aumentar el número de investigaciones, sería interesante replicar las estrategias de investigación ya desarrolladas para poder incrementar el grado de evidencia sobre su eficacia.

\section{Limitaciones del estudio}

Las conclusiones de esta revisión deben considerarse con cautela, puesto que los estudios obtenidos hasta 2019 fueron extraídos de las diferentes revisiones sistemáticas encontradas. En la presente revisión se establecieron unos criterios de inclusión y de exclusión, pero la mayoría de los estudios se seleccionaron de aquellos ya escogidos por cada revisión sistemática, y los cuales habían 
pasado ya por sus propios criterios de inclusión. Es posible que estudios que fueron excluidos por alguna revisión sistemática podrían haber sido seleccionados en nuestro estudio para su inclusión. No obstante, se espera que el presente estudio proporcione una guía útil sobre los niveles de evidencia de los principales tipos de intervenciones en comprensión lectora en autismo para profesionales de la educación e investigadores.

\section{Agradecimientos}

Este estudio está financiado por el Ministerio de Ciencia, Innovación y Universidades del Gobierno de España (PGC2018-096094-B-I00) y el V Plan Propio de Investigación y Transferencia de la Universidad de Sevilla (PPI2016-IV.5).

\section{Referencias $^{1}$}

American Psychiatric Association (APA) (2013). Diagnostic and statistical manual of mental disorders (DSM-5) (5th ed.). American Psychiatric Association.

*Arciuli, J., y Bailey, B. (2019). Efficacy of ABRACADABRA literacy instruction in a school setting for children with autism spectrum disorders. Research in Developmental Disabilities, 85, 114-115. https://doi.org/10.1016/j.ridd.2018.11.003

* Åsberg, J., y Sandberg, A. D. (2010). Discourse comprehension intervention for high-functioning students with autism spectrum disorders: preliminary findings from a school-based study. Journal of Research in Special Educational Needs, 10(2), 91-98. https://doi.org/10.1111/j.14713802.2010.01147.x

*Bailey, B., Arciuli, J., y Stancliffe, R. J. (2017). Effects of ABRACADABRA literacy instruction on children with autism spectrum disorder. Journal of Educational Psychology, 109(2), 257-268. https://doi.org/10.1037/edu0000138

*Bethune, K. S., y Wood, C. L. (2013). Effects of wh-question graphic organizers on reading comprehension skills of students with autism spectrum disorders. Education and Training in Autism and Developental Disabilities, 48(2), 236-244.

*Browder, D. M., Root, J. R., Wood, L., y Allison, C. (2017). Effects of a story-mapping procedure using the iPad on the comprehension of narrative texts by students with autism spectrum disorder. Focus on Autism and Other Developmental Disabilities, 32(4), 243-255. https://doi.org/10.1177/1088357615611387

Brown, H. M., Oram-Cardy, J., y Johnson, A. (2013). A meta-analysis of the reading comprehension skills of individuals on the autism spectrum. Journal of Autism and Developmental Disorders, 43 , 932-955. https://doi.org/10.1007/s10803-012-1638-1

*Carnahan, C. R., Williamson, P., Birri, N., Swoboda, C., y Snyder, K. K. (2016). Increasing comprehension of expository science text for students with autism spectrum disorder. Focus on Autism and Other Developmental Disabilities, 31(3), 208-220. https://doi.org/10.1177\%2F1088357615610539

Davidson, M. M., y Ellis Weismer, S. (2014). Characterization and prediction of early reading abilities in children on the autism spectrum. Journal of Autism and Developmental Disorders, 44(4), 828845. https://doi.org/10.1007/s10803-013-1936-2

*Drill, R. B., y Bellini, S. (2021). Combining readers theater, story mapping and video self-modeling interventions to improve narrative reading comprehension in children with autism spectrum

\footnotetext{
${ }^{1}$ Las referencias de los estudios primarios incluidos en la revisión aparecen marcadas con *
} 
disorder. Journal of Autism and Developmental Disorders, 1-178. https://doi.org/10.1007/s10803021-04908-X

El Zein, F., Solis, M., Vaughn, S., y McCulley, L. (2014). Reading comprehension interventions for students with autism spectrum disorders: A synthesis of research. Journal of Autism and Developmental Disorders, 44(6), 1303-1322. https://doi.org/10.1007/s10803-013-1989-2

*Flores, M. M., y Ganz, J. B. (2007). Effectiveness of direct instruction for teaching statement inference, use of facts, and analogies to students with developmental disabilities and reading delays. Focus on Autism and Other Developmental Disabilities, 22(4), 244-251. https://doi.org/10.1177/10883576070220040601

Gough, P. B., y Tunmer, W. E. (1986). Decoding, reading, and reading disability. Remedial and Special Education, 7(1), 6-10. https://doi.org/10.1177/074193258600700104

*Head, C. N., Flores, M. M., y Shippen, M. E. (2018). Effects of direct instruction on reading comprehension for individuals with autism or developmental disabilities. Education and Training in Autism and Developmental Disabilities, 53(2), 176-191.

Henderson, L. M., Clarke, P. J., y Snowling, M. J. (2014). Reading comprehension impairments in autism $\begin{array}{llll}\text { spectrum disorders. } & \text { L'Année } & \text { 779-797. }\end{array}$ https://doi.org/10.4074/S0003503314004084

Hoffmann, T. C., Glasziou, P. P., Boutron, I., Milne, R., Perera, R., Moher, D., Altman, D. G., Barbour, V., Macdonald, H., Johnston, M., Lamb, S. E., Dixon-Woods, M., McCulloch, P., Wyatt, J. C., Chan, A-W., y Michie, S. (2014). Better reporting of interventions: Template for intervention description and replication (TIDieR) checklist and guide. British Medical Journal, 348. https://doi.org/10.1136/bmj.g1687

Hoover, W. A., y Gough, P. B. (1990). The simple view of reading. Reading and Writing, 2(2), 127160. https://doi.org/10.1007/BF00401799

*Howorth, S., Lopata, C., Thomeer, M., y Rodgers, J. (2016). Effects of the TWA strategy on expository reading comprehension of students with autism. British Journal of Special Education, 43(1), 3959. https://doi.org/10.1111/1467-8578.12122

Hulme, C., y Snowling, M. J. (2011). Children's reading comprehension difficulties. Current Directions in Psychological Science, 20(3), 139-142. https://doi.org/10.1177/0963721411408673

*Jackson, E. M., y Hanline, M. F. (2020). Using a concept map with RECALL to increase the comprehension of science texts for children with autism. Focus on Autism and Other Developmental Disabilities, 35(2), 90-100. https://doi.org/10.1177/1088357619889933

*Kamps, D. M., Barbetta, P. M., Leonard, B. R., y Delquadri, J. (1994). Classwide peer tutoring: An integration strategy to improve reading skills and promote peer interactions among students with autism and general education peers. Journal of Applied Behavior Analysis, 27(1), 1297776. https://doi.org/10.1901/jaba.1994.27-49

*Kamps, D. M., Leonard, B., Potucek, J., y Garrison-Harrell, L. (1995). Cooperative learning groups in reading: An integration strategy for students with autism and general classroom peers. Behavioral Disorders, 21(1), 89-109. https://doi.org/10.1177\%2F019874299502100103

*Kamps, D., Locke, P., Delquadri, J., y Hall, R. V. (1989). Increasing academic skills of students with autism using fifth grade peers as tutors. Education and Treatment of Children, 12(1), 38-51.

Khowaja, K., y Salim, S. S. (2013). Review: A systematic review of strategies and computer-based intervention (CBI) for reading comprehension of children with autism. Research in Autism Spectrum Disorders, 7(9), 1111-1121. https://doi.org/10.1016/j.rasd.2013.05.009

*Kim, S. Y., Rispoli, M., Lory, C., Gregori, E., y Brodhead, M. T. (2018). The effects of a shared reading intervention on narrative story comprehension and task engagement of students with autism 
spectrum disorder. Journal of Autism and Developmental Disorders, 48(10), 3608-3622. https://doi.org/10.1007/s10803-018-3633-7

Kim, Y.-S. G. (2017). Why the simple view of reading is not simplistic: unpacking component skills of reading using a direct and indirect effect model of reading (DIER). Scientific Studies of Reading, 21(4), 310-333. https://doi.org/10.1080/10888438.2017.1291643

Kintsch, W. (1998). Comprehension: a paradigm for cognition. Cambridge University Press.

McIntyre, N. S., Oswald, T. M., Solari, E. J., Zajic, M. C., Lerro, L. E., Hughes, C., Devine, R. T., y Mundy, P. C. (2018). Social cognition and reading comprehension in children and adolescents with autism spectrum disorders or typical development. Research in Autism Spectrum Disorders, 54, 9-20. https://doi.org/10.1016/j.rasd.2018.06.004

Nation, K., y Norbury, C. (2005). Why reading comprehension fails: Insights from developmental disorders. Topics in Language Disorders, 25(1), 21-32.

Nation, K., Clarke, P., Wright, B., y Williams, C. (2006). Patterns of reading ability in children with autism spectrum disorder. Journal of Autism and Developmental Disorders, 36(7), 911-919. https://doi.org/10.1007/s10803-006-0130-1

*O'Connor, I. M., y Klein, P. D. (2004). Exploration of strategies for facilitating the reading comprehension of high-functioning students with autism spectrum disorders. Journal of Autism and Developmental Disorders, 34(2), 115-127. https://doi.org/10.1023/B:JADD.0000022603.44077.6b

*Reutebuch, C. K., El Zein, F., Kim, M. K., Weinberg, A. N., y Vaughn, S. (2015). Investigating a reading comprehension intervention for high school students with autism spectrum disorder: A pilot study. Research in Autism Spectrum Disorders, 9, 96-111. https://doi.org/10.1016/j.rasd.2014.10.002

*Roux, C., Dion, E., Barrette, A., Dupéré, V., y Fuchs, D. (2015). Efficacy of an intervention to enhance reading comprehension of students with high-functioning autism spectrum disorder. Remedial and Special Education, 36(3), 131-142. https://doi.org/10.1177/0741932514533998

*Singh, B. D., Moore, D. W., Furlonger, B. E., Anderson, A., Busacca, M. L., y English, D. L. (2017). Teaching reading comprehension skills to a child with autism using behaviour skills training. Journal of Autism and Developmental Disorders, 47(10), 3049-3058. https://doi.org/10.1007/s10803-017-3229-7

Solari, E. J., Grimm, R. P., McIntyre, N. S., Zajic, M., y Mundy, P. C. (2019). Longitudinal stability of reading profiles in individuals with higher functioning autism. Autism, 23(8), 1911-1926. https://doi.org/10.1177\%2F1362361318812423

*Solis, M., Reutebuch, C. K., Falcomata, T., Steinle, P. K., Miller, V. L., y Vaughn, S. (2021). Vocabulary and main idea reading intervention using text choice to improve content knowledge and reading comprehension of adolescents with autism spectrum disorder. Behavior Modification, 45(1), 66-98. https://doi.org/10.1177\%2F0145445519853781

*Stringfield, S. G., Luscre, D., y Gast, D. L. (2011). Effects of a story map on accelerated reader postreading test scores in students with high-functioning autism. Focus on Autism and Other Developmental Disabilities, 26(4), 218-229. https://doi.org/10.1177/1088357611423543

Tárraga-Mínguez, R., Gómez-Marí, I., y Sanz-Cervera, P. (2020). Interventions for improving reading comprehension in children with ASD: A systematic review. Behavioral Sciences, 11(1), 3. https://doi.org/10.3390/bs11010003

*Turner, H., Remington, A., y Hill, V. (2017). Developing an intervention to improve reading comprehension for children and young people with autism spectrum disorders. Educational and Child Psychology, 34(2), 13-26. 
Whalon, K. J., Al Otaiba, S., y Delano, M. E. (2009). Evidence-based reading instruction for individuals with autism spectrum disorders. Focus on Autism and Other Developmental Disabilities, 24(1), 316. https://doi.org/http://dx.doi.org/10.1177/1088357608328515

*Whalon, K., y Hanline, M. F. (2008). Effects of a reciprocal questioning intervention on the question generation and responding of children with autism spectrum disorder. Education and Training in Developmental Disabilities, 43(3), 367-387.

*Williamson, P., Carnahan, C. R., Birri, N., y Swoboda, C. (2015). Improving comprehension of narrative using character event maps for high school students with autism spectrum disorder. The Journal of Special Education, 49(1), 28-38. https://doi.org/10.1177/0022466914521301 


\section{Apéndice}

\section{Tabla}

Resumen de las intervenciones en comprensión lectora en personas con autismo

\begin{tabular}{|c|c|c|c|c|}
\hline \multicolumn{5}{|c|}{ Tipo de intervención: Instrucción de estrategias } \\
\hline Estudio & $\begin{array}{l}\text { Número de } \\
\text { Participantes }\end{array}$ & $\begin{array}{l}\text { Media } \\
\text { de } \\
\text { edad }\end{array}$ & Objetivos & Resultados \\
\hline $\begin{array}{l}\text { Arciuli y } \\
\text { Bailey } \\
(2019)\end{array}$ & 23 & $\begin{array}{l}7.13 \\
\text { años }\end{array}$ & $\begin{array}{l}\text { Aplicar el programa ABRACADABRA } \\
\text { para evaluar su eficacia en pequeños } \\
\text { grupos de niños con autismo en entorno } \\
\text { escolar. }\end{array}$ & $\begin{array}{l}\text { No se observaron mejoras en la } \\
\text { comprensión lectora. }\end{array}$ \\
\hline $\begin{array}{l}\text { Asberg y } \\
\text { Sandberg } \\
(2010)\end{array}$ & 12 & $\begin{array}{c}13 \\
\text { años }\end{array}$ & $\begin{array}{l}\text { Examinar la influencia de la estrategia } \\
\text { Pregunta-Respuesta (QAR) en el } \\
\text { rendimiento de la comprensión lectora. }\end{array}$ & $\begin{array}{l}\text { Mejoras significativas en la } \\
\text { comprensión del discurso hasta } \\
\text { cuatro semanas después del } \\
\text { entrenamiento. }\end{array}$ \\
\hline $\begin{array}{l}\text { Bailey et al. } \\
\qquad(2017)\end{array}$ & 20 & $\begin{array}{c}7.4 \\
\text { años }\end{array}$ & $\begin{array}{l}\text { Aplicar el programa de lectoescritura } \\
\text { asistido por ordenador } \\
\text { ABRACADABRA para mejorar la } \\
\text { alfabetización, la fluidez lectora, la } \\
\text { comprensión lectora y la escritura. }\end{array}$ & $\begin{array}{l}\text { Mejoras en la precisión y } \\
\text { comprensión lectora. }\end{array}$ \\
\hline $\begin{array}{l}\text { Bethune y } \\
\text { Wood } \\
(2013)\end{array}$ & 3 & $\begin{array}{l}9.3 \\
\text { años }\end{array}$ & $\begin{array}{l}\text { Evaluar los efectos de los organizadores } \\
\text { gráficos para responder preguntas. }\end{array}$ & $\begin{array}{l}\text { Mayor precisión en las respuestas } \\
\text { a las preguntas tras la intervención } \\
\text { y en el mantenimiento. }\end{array}$ \\
\hline $\begin{array}{l}\text { Browder et } \\
\text { al. (2017) }\end{array}$ & 3 & 9 años & $\begin{array}{l}\text { Evaluar los efectos de una intervención } \\
\text { que usa mapas de historias digitales en } \\
\text { iPads con cuatro estrategias para la } \\
\text { comprensión: organizadores gráficos, } \\
\text { estructura de la historia, respuesta a } \\
\text { preguntas e instrucción de estrategias } \\
\text { múltiples. }\end{array}$ & $\begin{array}{l}\text { El uso de los mapas de historias } \\
\text { resultó efectivo para la } \\
\text { comprensión lectora. }\end{array}$ \\
\hline $\begin{array}{l}\text { Carnahan et } \\
\text { al. (2016) }\end{array}$ & 3 & $\begin{array}{l}15.7 \\
\text { años }\end{array}$ & $\begin{array}{l}\text { Evaluar los efectos de la instrucción en } \\
\text { identificación de estructuras de textos } \\
\text { utilizando organizadores gráficos en la } \\
\text { comprensión de textos científicos } \\
\text { tradicionales. }\end{array}$ & $\begin{array}{l}\text { La instrucción fue altamente } \\
\text { efectiva durante las fases de } \\
\text { intervención y mantenimiento. }\end{array}$ \\
\hline $\begin{array}{l}\text { Stringfield et } \\
\text { al. (2011) }\end{array}$ & 3 & 9 años & $\begin{array}{l}\text { Usar mapas de historias como } \\
\text { organizador gráfico para aumentar la } \\
\text { comprensión lectora. }\end{array}$ & $\begin{array}{l}\text { Los mapas de historias mejoraron } \\
\text { la comprensión lectora de forma } \\
\text { rápida y se mantuvo durante todo } \\
\text { el estudio. }\end{array}$ \\
\hline $\begin{array}{l}\text { Whalon y } \\
\text { Handline } \\
\text { (2008) }\end{array}$ & 3 & $\begin{array}{c}8.1 \\
\text { años }\end{array}$ & $\begin{array}{l}\text { Utilizar preguntas recíprocas y el plan de } \\
\text { estudios SCORE (compartir ideas, } \\
\text { complementar a los demás, ofrecer } \\
\text { ayuda, recomendar cambios y ejercer } \\
\text { autocontrol). }\end{array}$ & $\begin{array}{l}\text { Aumento de la generación de } \\
\text { preguntas y respuestas, con } \\
\text { mejora en la comprensión lectora. }\end{array}$ \\
\hline $\begin{array}{l}\text { Williamson } \\
\text { et al. }(2015)\end{array}$ & 3 & $\begin{array}{l}16.3 \\
\text { años }\end{array}$ & $\begin{array}{l}\text { Evaluar la efectividad de un paquete de } \\
\text { intervención para aumentar la } \\
\text { comprensión de textos narrativos. }\end{array}$ & $\begin{array}{l}\text { Aumento inmediato en la } \\
\text { comprensión lector, la cual se } \\
\text { mantuvo alta en la intervención y } \\
\text { en el seguimiento. }\end{array}$ \\
\hline \multicolumn{5}{|c|}{ Tipo de intervención: Indicación anafórica } \\
\hline Estudio & $\begin{array}{l}\text { Número de } \\
\text { Participantes }\end{array}$ & $\begin{array}{l}\text { Media } \\
\text { de } \\
\text { edad }\end{array}$ & Objetivos & Resultados \\
\hline
\end{tabular}




\begin{tabular}{|c|c|c|c|c|}
\hline $\begin{array}{l}\text { O’Connor y } \\
\text { Klein (2004) }\end{array}$ & 20 & $\begin{array}{l}15.1 \\
\text { años }\end{array}$ & $\begin{array}{l}\text { Examinar los efectos que podían tener la } \\
\text { identificación de anáforas (entre ellos } \\
\text { pronombres). }\end{array}$ & $\begin{array}{l}\text { Efectos positivos y significativos } \\
\text { de la señalización anafórica en la } \\
\text { compresión lectora. }\end{array}$ \\
\hline \multicolumn{5}{|c|}{ Tipo de intervención: Instrucción explícita } \\
\hline Estudio & $\begin{array}{l}\text { Número de } \\
\text { Participantes }\end{array}$ & $\begin{array}{l}\text { Media } \\
\text { de } \\
\text { edad }\end{array}$ & Objetivos & Resultados \\
\hline $\begin{array}{c}\text { Flores y } \\
\text { Ganz (2007) }\end{array}$ & 4 & $\begin{array}{c}12 \\
\text { años }\end{array}$ & $\begin{array}{l}\text { Utilizar el programa de instrucción } \\
\text { directa Pensamiento Correctivo de } \\
\text { Lectura Básico: nivel A de comprensión. }\end{array}$ & $\begin{array}{l}\text { Aumento de las respuestas } \\
\text { correctas en las pruebas de } \\
\text { habilidades de comprensión } \\
\text { lectora hasta un mes después de la } \\
\text { intervención. }\end{array}$ \\
\hline $\begin{array}{l}\text { Head et al. } \\
\qquad(2018)\end{array}$ & 3 & $\begin{array}{l}13.3 \\
\text { años }\end{array}$ & $\begin{array}{l}\text { Aplicación del programa de } \\
\text { Comprensión de Lectura Correctiva: B1. }\end{array}$ & $\begin{array}{l}\text { Dominación de las habilidades } \\
\text { específicas de comprensión } \\
\text { lectora rápidamente y, } \\
\text { mantenimiento y generalización } \\
\text { tras la aplicación del programa. }\end{array}$ \\
\hline $\begin{array}{l}\text { Howorth et } \\
\text { al. (2016) }\end{array}$ & 4 & $\begin{array}{l}10.5 \\
\text { años }\end{array}$ & $\begin{array}{l}\text { Examinar el efecto de la estrategia de } \\
\text { pensar antes de leer, pensar mientras se } \\
\text { lee y pensar después de leer (TWA) en la } \\
\text { comprensión de textos expositivos. }\end{array}$ & $\begin{array}{l}\text { La TWA mejoró la comprensión } \\
\text { lectora de los textos expositivos } \\
\text { después de la intervención y en el } \\
\text { seguimiento. }\end{array}$ \\
\hline
\end{tabular}

\begin{tabular}{|c|c|c|c|}
\hline \multicolumn{4}{|r|}{ Tipo de intervención: Grupo de estudiantes } \\
\hline Estudio & $\begin{array}{c}\text { Número de } \\
\text { Participantes }\end{array}$ & $\begin{array}{l}\text { Media } \\
\text { de } \\
\text { edad }\end{array}$ & Objetivos \\
\hline $\begin{array}{c}\text { Kamps et al. } \\
\text { (1989) }\end{array}$ & 2 & $\begin{array}{c}10 \\
\text { años }\end{array}$ & $\begin{array}{l}\text { Usar la estrategia de tutoría entre pares } \\
\text { para mejorar la comprensión lectora } \\
\text { (alumno neurotípico y alumno con } \\
\text { TEA). }\end{array}$ \\
\hline $\begin{array}{l}\text { Kamps, et } \\
\text { al. (1994) }\end{array}$ & 3 & $\begin{array}{c}8.3 \\
\text { años }\end{array}$ & $\begin{array}{l}\text { Usar la estrategia de tutoría entre pares } \\
\text { para mejorar la comprensión lectora } \\
\text { (alumno neurotípico y alumno con } \\
\text { TEA). }\end{array}$ \\
\hline $\begin{array}{l}\text { Kamps, et } \\
\text { al. (1995) }\end{array}$ & 3 & $\begin{array}{c}11 \\
\text { años }\end{array}$ & $\begin{array}{l}\text { Usar grupos de aprendizaje cooperativo } \\
\text { para promover las habilidades de lectura } \\
\text { de los estudiantes con autismo y } \\
\text { neurotípicos. }\end{array}$ \\
\hline $\begin{array}{l}\text { Reutebuch et } \\
\text { al. (2015) }\end{array}$ & 3 & $\begin{array}{c}16 \\
\text { años }\end{array}$ & $\begin{array}{l}\text { Emparejar cada estudiante con TEA con } \\
\text { otro neurotípico para aprender y usar } \\
\text { estrategias de lectura en textos } \\
\text { informativos y valorar otras variables } \\
\text { (comportamiento desafiante e interacción } \\
\text { social). }\end{array}$ \\
\hline
\end{tabular}

Tipo de intervención: Combinada

\begin{tabular}{|c|c|c|c|c|}
\hline Estudio & $\begin{array}{l}\text { Número de } \\
\text { Participantes }\end{array}$ & $\begin{array}{l}\text { Media } \\
\text { de } \\
\text { edad }\end{array}$ & Objetivos & Resultados \\
\hline $\begin{array}{l}\text { Drill y } \\
\text { Bellini } \\
(2021)\end{array}$ & 3 & $\begin{array}{c}13 \\
\text { años }\end{array}$ & $\begin{array}{l}\text { Evaluar la eficacia de un paquete de } \\
\text { intervención que combina teatro de } \\
\text { lectores, mapas de historias y } \\
\text { automodelado por vídeo. }\end{array}$ & $\begin{array}{l}\text { El paquete de intervención mejoró } \\
\text { la comprensión lectora. }\end{array}$ \\
\hline
\end{tabular}




\begin{tabular}{|c|c|c|c|c|}
\hline $\begin{array}{l}\text { Jackson y } \\
\text { Handline } \\
\text { (2020) }\end{array}$ & 2 & 5 años & $\begin{array}{l}\text { Evaluar la intervención de lectura } \\
\text { compartida RECALL combinada con el } \\
\text { uso de mapas conceptuales para } \\
\text { responder preguntas de comprensión de } \\
\text { textos científicos. }\end{array}$ & $\begin{array}{l}\text { La intervención resultó efectiva } \\
\text { para aumentar las respuestas } \\
\text { correctas de los participantes a las } \\
\text { preguntas de comprensión de } \\
\text { textos científicos. }\end{array}$ \\
\hline $\begin{array}{c}\text { Kim et al. } \\
\text { (2018) }\end{array}$ & 3 & 7 años & $\begin{array}{l}\text { Evaluar los efectos de la intervención de } \\
\text { lectura compartida en la comprensión de } \\
\text { la historia narrativa y la participación en } \\
\text { tareas de lectura. }\end{array}$ & $\begin{array}{l}\text { Mejoras notables en comprensión } \\
\text { lectora y participación en tareas de } \\
\text { lectura tanto tras la aplicación de } \\
\text { una intervención como en los } \\
\text { datos de mantenimiento. }\end{array}$ \\
\hline $\begin{array}{l}\text { Roux et al. } \\
\text { (2015) }\end{array}$ & 45 & 9 años & $\begin{array}{l}\text { Valorar la eficacia de la instrucción } \\
\text { explícita en diferentes habilidades } \\
\text { esenciales para la comprensión de textos } \\
\text { (conocimiento del significado de } \\
\text { palabras o vocabulario, identificación de } \\
\text { la idea principal, estructuración del texto } \\
\text { y relaciones anafóricas). }\end{array}$ & $\begin{array}{l}\text { Aumento de su comprensión } \\
\text { lectora, conocimiento del } \\
\text { significado de más palabras y } \\
\text { capacidad de identificar ideas } \\
\text { principales y relaciones } \\
\text { anafóricas. }\end{array}$ \\
\hline $\begin{array}{l}\text { Turner et al. } \\
\text { (2017) }\end{array}$ & 29 & $\begin{array}{l}13.5 \\
\text { años }\end{array}$ & $\begin{array}{l}\text { Aplicación de un programa de } \\
\text { Enseñanza Recíproca (cuatro estrategias: } \\
\text { predecir, aclarar, cuestionar y resumir, } \\
\text { las cuales se modelan mientras el alumno } \\
\text { lee). }\end{array}$ & $\begin{array}{l}\text { El grupo que recibió la } \\
\text { intervención mejoró } \\
\text { significativamente en } \\
\text { comprensión lectora. }\end{array}$ \\
\hline $\begin{array}{l}\text { Singh et al. } \\
\quad(2017)\end{array}$ & 1 & $\begin{array}{c}13 \\
\text { años }\end{array}$ & $\begin{array}{l}\text { Aplicación de un programa de } \\
\text { Enseñanza Recíproca (instrucción, } \\
\text { modelado, ensayo y retroalimentación). }\end{array}$ & $\begin{array}{l}\text { Mayor rendimiento del } \\
\text { participante en cada habilidad de } \\
\text { comprensión lectora. }\end{array}$ \\
\hline $\begin{array}{l}\text { Solis et al. } \\
\text { (2021) }\end{array}$ & 5 & $\begin{array}{l}13.6 \\
\text { años }\end{array}$ & $\begin{array}{l}\text { Investigar los efectos de una } \\
\text { intervención multicomponente de } \\
\text { vocabulario y lectura y que incorpora un } \\
\text { componente de elección (texto). }\end{array}$ & $\begin{array}{l}\text { La intervención se asoció con } \\
\text { mejores resultados de los } \\
\text { estudiantes en las medidas } \\
\text { utilizadas para evaluar la } \\
\text { comprensión lectora y el } \\
\text { vocabulario basadas en el plan de } \\
\text { estudios. }\end{array}$ \\
\hline
\end{tabular}

